\title{
Brain-brain interface tested in rat 'mind-meld'
}

In a story that might have come from science fiction, neuroscientists at Duke University (Durham, NC) directly linked the brains of two rats, enabling them to share information. Once trained in a simple task-pressing a lever corresponding to an indicator light or correctly identifying an opening in a barrier as wide or narrow in order to obtain a reward-pairs of rats were connected via implanted microelectrode arrays and placed in separate cages.

Only one of the rats, the 'encoder,' was exposed to the stimulus (the indicator light or the opening), and upon completion of the task, its brain activity was recorded and transmitted to the other, 'decoder', rat. The decoder rat then had to select the correct response based solely on the information it received from the encoder rat. The decoder rats achieved success rates of $\sim 70 \%$ in the indicator light test and $\sim 65 \%$ in the opening width test (Sci. Rep. 3, 1319; 2013).

The scientists even paired two rats located on different continents, transmitting the brain signal over the internet. Although the

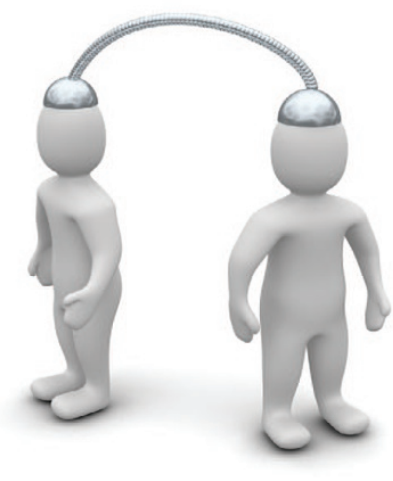

was not associated with the project, told New Scientist that it's unclear exactly what information is being communicated between the rats. The experiments, he said, "blast[ed] a relatively large area of the brain with a signal they're not sure is $100 \%$ correct. We are still using a sledgehammer to crack a walnut." Andrew Schwartz (a neurobiologist at University of Pittsburgh, PA) noted in a Nature News story that the decoder rats performed relatively poorly given a basic task with only two choices and a $50 \%$ success rate expected by chance. Lee Miller (a physiologist at Northwestern University, Evanston, IL) told Nature News, "It is not clear to what end the effort is really being made." Sliman Bensmaia, a neuroscientist from the University of Chicago (IL), added that if Nicolelis's goal was to improve neural prosthetics, then "the design seems convoluted and irrelevant," and if the goal was to build a computer, then "the proposition is speculative and the evidence underwhelming."

Monica Harrington

\section{IN BATTLE OF THESEXES, FOXP2 UNDERLIIES COMMUNICATION ADVANTAGE}

Foxp2 protein, which regulates the expression of a large number of genes in the brain, is involved with speech and language in humans and with vocal communication in other mammals and birds. Mutations in human FOXP2 result in impaired language and speech, and birds with reduced Foxp2 expression have difficulty imitating the songs of their tutors. Researchers at the University of Maryland School of Medicine, Baltimore, showed that male rats have more Foxp2 protein than females in areas of the brain associated with cognition, emotion and vocalization (J. Neurosci. 33, 3276-3283; 2013).

The team of scientists, led by Margaret M. McCarthy, also showed the opposite was true for humans: females showed higher basal levels of FOXP2 than males in the cortex, specifically in an area of the left hemisphere thought to be involved in language. Studies have shown that girls tend to speak earlier and with greater complexity than boys of the same age.

To determine whether the difference in levels of Foxp2 in the rat males and females affected communication, the scientists recorded and quantified the ultrasonic distress calls of 4-d-old pups that were separated from the dam for 5 min. The male pups averaged twice as many calls as the females. When the researchers used small interfering RNA (siRNA) to reduce levels of the Foxp2 protein in the male pups, they produced significantly fewer vocalizations than control males. Unexpectedly, siRNA-treated females produced significantly more vocalizations than control females. The authors determined that the siRNA acted differently in females than in males, decreasing Foxp2 levels in males but ultimately doubling them in females after an initial decrease. Therefore, in both rats and humans, higher levels of Foxp2 expression were found in the more communicative sex.

Not only did the siRNA treatment reverse the sex difference in the quantity of distress calls, but it also reversed the difference in the quality of the vocalizations. Males typically vocalized at a lower frequency and higher amplitude than females, but siRNA-treated females produced calls lower in frequency and higher in amplitude than siRNA-treated males.

Next, the scientists wanted to see if the difference in vocalizations had any functional consequences. As they predicted, the dam retrieved the male pups first and returned them to the nest first. siRNA-treated females were retrieved before control females, and control males were retrieved before siRNA-treated males, demonstrating that the vocalizations directly influenced the dam's retrieval behavior. 